\title{
User research in a scientific software development project
}

\author{
David Sloan, Catriona Macaulay, Paula Forbes, Scott Loynton, Peter Gregor \\ School of Computing \\ University of Dundee \\ Dundee, Scotland, UK \\ +44 1382385598
}

\{dsloan, catriona, pforbes, scottloynton, pgregor\}@computing.dundee.ac.uk

\begin{abstract}
The Usable Image project provides usability and user-centred design support to a scientific software development project. OMERO is a complex software application aimed at supporting the management, analysis and processing of microscopy images and associated data. In order to gather a richer understanding of the diversity and similarities of scientific practice and the role technology plays in supporting the work of scientists using images and image-related data, a range of user-research techniques have been applied, including design ethnography and surveys. This work has provided insights that have informed the development team, increasing knowledge and understanding of what is a complex usage environment, and helping in the process of creating a more usable and useful scientific tool.
\end{abstract}

This paper discusses the insights gained from the ethnographic work and from user surveys, in terms of attitudes to and usage patterns of technology amongst life science researchers, and considers the implications of these insights on the user-centred design and development of OMERO.

\section{Categories and Subject Descriptors}

H.5.2 User Interfaces (D.2.2, H.1.2, I.3.6).

\section{General Terms}

Management, Measurement, Documentation, Design, Human Factors, Standardization.

\section{Keywords}

Scientific software, usability, user-centred design, design ethnography

\section{INTRODUCTION}

Successful technological innovation in a scientific content involves not only the ability gather and analyse larger and more diverse quantities of data in new ways, but also to improve efficiency and effectiveness of existing tasks [2]. Visualising large

(C) The Authors 2009.

Published by the British Computer Society sets of data in a way that enhances the quality of analysis that can be performed on it is an ongoing usability challenge for scientific software [3]. Given the complexity and diversity of the problem space - scientific laboratories in which varied but cognitively demanding workflows take place in high pressure working environments, the usability challenges facing a scientific software development project are not insignificant [7].

In this paper we discuss the Usable Image project's work in promoting user-centred design of OMERO, a scientific software system aimed at enabling biological microscopy data management and analysis. In particular, we focus on ethnographic and survey activities in gaining a rich understanding of the diversity of user and usage environment, and how this insight has influenced the software development process.

\section{BACKGROUND}

\subsection{Tackling the challenge of creating usable scientific software}

Much scientific software development is carried out by end users - by scientists who create programs to support their investigations. Letondal and Mackay [9] investigated scientific computer tool usage in the Institut Pasteur in Paris and identified distinct groups of users based on their level of involvement in configuring the software they use in their work This means that software may be developed by users who have not had formal training in testing - whether technical or usability testing - nor who may have the time, expertise or inclination to adopt usercentred design methods; Sanders and Kelly [14] interviewed 16 scientific software developers and users and found $75 \%$ had not undergone any formal training in software engineering. The open source nature of much scientific software development may also lead to a focus on extending functionality at the expense of usability for an audience beyond the small team of developerusers [13].

Where scientific software development is not being carried out by scientists themselves, the complexity of the problem space and requirements definition may be particularly challenging - given that in a research context the goals in question are potentially unknown, beyond some form of 'discovery'. Letondal and Mackay [9] promoted 'participatory programming' through a series of sessions encouraging scientists to share stories of how they might use a software application, in an effort to support the development of software that can be tailored for their needs. In this way, they were able to identify effective ways for developers and scientists to work together on refining generic requirements and areas where personalisation was necessary. In their Making 
Tea project, Schraefel et al used the power of analogy in an effort to achieve mutual understanding between developers and scientists of the problem space and apply an appropriate technological intervention [16].

\subsection{The Open Microscopy Environment and OMERO}

The Open Microscopy Environment (OME) Consortium is a group of biologists and software developers who are working together to produce a range of open source informatics solutions for the storage and analysis of optical microscope image data [6]. A keystone of this development work is OMERO, a suite of server-client technologies to provide scientists with a system for managing, analysing, and sharing light microscopy images and associated scientific data generated throughout the scientific process.

Crucially, OMERO has been designed to be file-agnostic, allowing management and viewing of images of a variety of file formats and acquisition sources together in one place, avoiding the need for proprietary software solutions currently required to view images captured by a particular microscope. As such, much of the developmental effort has been in developing a robust clientserver architecture to enable processing of large quantities of data, and enable multiple users to efficiently store, retrieve and manage their data. For scientists, the main OMERO application is a Java desktop client, allowing:

- Importing of microscopy data, in a range of formats proprietary and open - into the OMERO image format;

- Management of images and associated metadata;

- Visualisation of images, in multiple dimensions: typically $\mathrm{x}, \mathrm{y}$, $\mathrm{z}$ and across time, plus the ability to view individual wavelength channels;

- Basic measurement - including lengths and pixel intensity values of areas;

- Projection of images across a specific dimension;

- Saving images and parts of images in generic file formats, for example to illustrate publications.

- Outputting of reports of specific queries or views of data.

Other developments have included a web based interface to OMERO, allowing access to and management of images via a browser, without requiring the OMERO client application to be installed, thus significantly enhancing the ability to share the data. Supporting this has been the development of a tool to create, manage and share experimental protocols - allowing images and related data to be associated with the protocol used to generate them. In this way, OMERO functionality has been extended to provide what is effectively a simple electronic lab-book, a longtime focus of activity in scientific software development [16].

While OMERO meets definitions of scientific software [14] in that it takes as input raw data acquired by a microscope, processes this data and enables novel visualisation possibilities for this multi-dimensional data in order to support analysis of biological specimens, there is also a more generic aspect to the software. This is its aim in supporting collaborative working, through providing users with the ability to share data, browse colleagues' data, and offer opinion and advice through for example annotations and tags. In this way, OMERO aims to support reusability of data over time, by storing contextual information about images, such as the experimental protocol used to generate the image, or details of the subject of the image, such that even if the scientist who captured the image is no longer available, the lab can continue to use the data at a later date.

\subsection{The Usable Image Project}

After over two years of development activity, it was recognised by the OMERO team that there was a need to supplement the team's domain and technical expertise with additional user-centred input, ranging from gaining a better understanding of user diversity to specific usability evaluation techniques. The aim would be on the one hand to optimise the usability of the software as it evolves, but also to feed in new requirements based on user research in order to increase OMERO's marketability to new users. As a result, the Usable Image team, a multi-disciplinary group including design ethnographers and usability/interaction design specialists, was established to support the OMERO development team, while also investigating novel approaches to user-centred design in a complex environment.

The Usable Image (UI) project is a three-year UK EPSRC funded research project investigating ways of integrating user-centred system design approaches [7] into scientific software development, and OMERO affords it a real-world scientific software development project as a case study.

The Usable Image team's role within the OME is therefore to support the development of OMERO by providing expertise in a range of user-centred design techniques, each aimed at understanding more about the users and usage environment of the software in development, how best the software can support the users' needs, and how well the software currently meets these needs. After an initial period of familiarisation with the software development process and lifecycle, a methodology was adopted to apply a selection of UCD techniques at appropriate stages in the lifecycle in order to provide the development team with enhanced information about the target audience and the suitability of the software to their needs.

\section{USER RESEARCH METHODOLOGY}

The Usable Image team was faced with the task of promoting UCD in an already mature software development project, which had adopted an Agile-style development approach [1] of regular and frequent iterations of the system in development, following an Open Source Software (OSS) philosophy of making the codebase freely available to others who wished to contribute to development.

Added to this was the location of the development team - the majority of whom work in a life science research centre, managed by a biology professor, and therefore with a readily accessible but limited - source of user requirements on which to base development. The challenge was therefore to widen knowledge of user characteristics and usage environments in a way that could inform development and enhance the quality of the software developed. An approach combining a range of user-centred design activities was taken, including activities grounded in the social sciences, in particular ethnography, focusing on understanding the scientists and their work, to more conventional user-centred design activities focusing on evaluating the usability of the 
software itself - including expert usability inspections and user observations Error! Reference source not found.

In the following sections we discuss the impact of two specific UCD activities we have undertaken, and how this information has informed other activities and the development of the software itself. We concentrate particularly on the development of knowledge of scientific workflow, and involvement of and attitudes to technology in supporting work.

\section{ETHNOGRAPHIC STUDIES}

As a means of informing design through a more holistic understanding of a system's current and potential users and usage environment and context, design ethnography has emerged as a valuable user research tool [10]. The Usable Image team's ethnographer spent 18 months conducting ethnographic observations of scientists in two research institutions. Over this period of time, more than 100 observations were conducted of over 60 scientists.

These observations took place over periods of time ranging from 30 minutes to full days, mainly focusing on the work of an individual scientist, but also on occasion group activities, such as lab meetings or more informal social gatherings away from the lab. Notes were taken by hand; but audio and video recordings supplemented observations where activities became particularly complex, or where there was a particular need to record artifacts of the activity.

Observations were anonymised, written up into short illustrated stories, and shared with the rest of the Usable Image team for initial analysis and reflection. Analysis involved coding each story using a collaboratively evolving set of thematic codes, leading to a number of user stories, which captured key issues or emerging themes. These stories were also published on the project wiki, and made available to the development team, who were encouraged to read and react to the stories by identifying areas requiring further clarification.

\section{SURVEYS}

Balancing the rich data gathered by the ethnographic stories, a series of surveys was conducted of life scientists as potential users. Since a key goal of OMERO is to support data sharing and collaboration in scientific activity beyond the physical and virtual boundaries of discrete research organisations, the need to attract usage across diverse geographical and cultural locations was essential in order to avoid the system being confined to a specific group of people with specific needs.

In particular, we were keen to find out how life scientists approach the task of capturing, storing and analysing biological images. We developed a series of three Web based surveys, and invited participation through emails to relevant email discussion lists. The surveys were run in series, and refinement of questions took place after each survey, in order to focus on areas of attention most relevant to the software development process the Usable Image team was supporting.

Two of the surveys were geographically and organisationally constrained to specific research institutions; Survey $1(n=41)$ took place in the same institution in which the ethnographic and usability testing activities took place; Survey $2(n=35)$ was conducted in another research institution in Europe. Survey 3 $(n=34)$ was open to all respondents involved in using microscopy imaging as part of their work; invitations for this list were sent to email lists specializing in microscopy imaging.

The survey questions were drafted by a sociologist and an interaction designer, in consultation with imaging software developers, life scientists, and scientific officers. As the surveys were primarily aimed at gathering information relevant to the development of the OMERO software, an iterative process was adopted whereby the results of the first and second surveys were analysed for key trends, and in particular for areas where answers provided an insufficient level of detail for the OMERO developers. This information, in conjunction with open questions emerging from other Usable Image team activities involving gathering feedback from scientists, was used to define questions for the third survey, exploring in more detail relevant areas requiring investigation.

The majority of questions were multiple-choice; some were freetext choices or some are purely free-text answers. Participation was voluntary and anonymous; each survey was designed to be completed in 15-20 minutes. The surveys were administered online, using the SurveyMonkey survey creation and management tool (http://www.surveymonkey.com).

\section{INSIGHTS GATHERED}

Based both on our in-situ ethnographic activity and remote interaction with scientists through the series of surveys, we have gathered an enhanced understanding of technology usage and involvement amongst scientists who work with light microscopy images; and how it shapes and influences activity. While acknowledging there are limitations in generalisability of the data gathered from both activities, we have been able to make the following observations, each of which has proved valuable in informing and influencing development direction in the particular context of the development of open source scientific software for use by scientists. In some cases our observations may reinforce existing knowledge; in other cases we may be able to contribute novel insights to a complex development environment.

\subsection{Diversity of technology used}

We found that scientists working with microscopy data use a variety of tools in the typical workflow of experiment preparation, image acquisition, manipulation, analysis and output for publication; the acquisition, manipulation and analysis stages often being repeated several times during the process. In addition to dedicated software, for example to control the acquisition process at the microscope, or to process the images to a point that they are ready for analysis, scientists also use generic graphic design, spreadsheet and presentation software.

Our ethnographic stories revealed the diversity of software used, and gave an insight into the extent to which the scientist used the range of functionality of the software - frequently only an extremely small subset of a very complex and powerful application. We followed this up with more directed investigation through our surveys, which revealed:

- A wide range of software applications was used - participants $(n=41)$ named 24 separate software applications.

- The mean number of software applications used by scientists for image analysis was 3.5 . 
- There was a mix of dedicated and specialist scientific software applications alongside generic desktop applications, the most popular of which for image analysis were Photoshop (68\%), but generic spreadsheet and presentation software (Excel and PowerPoint) were also extremely widely used.

\subsection{Workflow rigidity}

Several ethnographic stories focused on a 'day in the life' of a scientist; which gave opportunity to focus on the extent to which routine tasks were performed as part of an experiment, and where deviations from standard protocols took place. While experiments typically followed a generic protocol, there were frequent periods where the next step depended very much on a visual inspection of a sample, or of data gathered - often to investigate 'interesting' observations noted on certain images. Deviations from standard protocols were identified in different ways - hand annotations of printouts, notes in lab books, post-it notes.

\subsection{Interpretation of key concepts: 'image' and 'metadata'}

Earlier surveys and ethnographic studies indicated that there was a range of interpretation of two key concepts of OMERO: the 'image' and 'metadata'. Both of these were fundamental to the underlying architecture of the software, and the user interface, in terms of what data should be shown, how, and when; and the development team had very specific technical definitions of the terms. For the term 'image', we had assumed a working definition of a visual representation of the biological sample, acquired by the microscope; and other information associated with that image we referred to as 'metadata'. However, we became aware that as scientists worked with images of different dimensions, there was not unanimous agreement over the concept of an 'image'; further, it became apparent that there was no overwhelming familiarity with the term 'metadata'. This caused some frustration amongst developers when they realised their definitions were not universally shared.

In Survey $3(n=34)$, we specifically asked people to define, in their own words, what they understood by the two terms. A simple content analysis of answers illustrated the diversity of responses.

In defining the term 'image', definitions tended to focus on describing characteristics/attributes of the image, and also where it came from and what one could or would do with it:

- $24 \%$ mentioned dimensions, but there was no consensus as to whether an image consisted of two, three or more dimensions.

- $36 \%$ mentioned 'pixels'; $14 \%$ mentioned 'numbers' and $14 \%$ mentioned 'data.'

- $41 \%$ mentioned the purpose of the image (i.e. why it had been captured); $18 \%$ mention acquisition details; and $12 \%$ mentioned the subject being imaged.

- $24 \%$ gave non-specific or circular definitions.

When we asked for descriptions of the term 'metadata', we asked respondents to consider the format, nature, and acquisition of the information in question.

- $18 \%$ mentioned format in their description - the vast majority of whom indicated metadata could be digital or non-digital, while 2 respondents indicated it was digital information only.
- $32 \%$ mentioned acquisition in their description; of these $29 \%$ indicated metadata could be captured automatically or digitally.

- $41 \%$ gave non-specific descriptions, nearly half of whom explicitly indicated they were not familiar with the term.

\subsection{Attitudes to sharing and collaboration}

We might expect that the nature of scientific data - data gathered during an experiment - would be jealously guarded by scientists, and shared only with trusted colleagues, such as a supervisor, until the point of publication. Ethnographic stories revealed informal collaboration, often in an effort to provide a second opinion, or to attempt to error-trace, or for validation of quality, but beyond this there was less evidence of a desire to be able to share data more widely, except where this might involve looking at archive data (digital and paper-based notes).

Our surveys asked scientists about when and with whom they shared information; and overwhelmingly scientists did share their own data with others: supervisor, colleagues within research group, colleagues outside the scientist's own research group, and even colleagues external to the scientist's research institution. It is likely, though not revealed by the survey, that these shares would be user-instigated - i.e. requested by the user, rather than

\subsection{Dealing with problems with technology}

One area of interest that emerged during the ethnographic work was the unstructured way in which scientists acquired skills in ICT use necessary for them to do their job - or improve efficiency, or allow them to explore new avenues of discovery and related to this was how they managed situations where they did not have sufficient skills to diagnose and fix a technology related problem.

Several stories focused on the frustrations of working with microscopes - complex and expensive pieces of technology that required booking in advance, and were available for limited periods of time. This meant that scientists would be required to plan experimental design around the availability of a microscope, meaning careful advance gathering and preparation of all samples. As complex instruments, microscopes required some degree of familiarisation, and could be unreliable either through malfunction or settings changed by a previous user.

Stories focused on where a problem with the microscope could lead to significant disruption, and hence stress, amongst scientists. Specialist support able to diagnose and remedy faults was not always available at the time the scientist required it; and so we gained an insight into what a scientist might do in order to address a problem with the microscope - ask a lab-mate present in the building; ask a colleague who happened to be passing by, but who may not be known well to them. Over time, scientists might be unwilling to return to a particular source of help, for fear of being seen to be a nuisance, and as a result might adjust their working practice to avoid the problem.

One story tells of a situation where a newly updated microscope caused a scientist extreme frustration as she sought to identify what was wrong while being fearful of causing further problems by changing settings unnecessarily. After failing to find help in the research institution, she reluctantly telephoned her lab leader 
on a Saturday evening. After much effort he found an unplugged cable which, when reattached, solved the problem, but caused a significant delay in the scientist's work.

Letondal and Mackay [9] described how the profile of scientists in terms of proactivity in acquiring and configuring software tools to support their work has changed, as younger scientists become more proficient in procuring and customising solutions for their needs. This may be so, but in our ethnographic stories we found several examples of scientists of a similar age and status 'who just want to be able to get on with their work' without spending excessive time developing skills in a particular piece of software, even if it might improve workflow efficiency longer term.

Survey 2 asked participants about their strategies for dealing with problems encountered when using software. The most common response $(n=30)$ was to ask a colleague with expertise for advice (30\% (9) respondents would always take this action, 50\% (15) would usually do this), while secondary strategies included searching for training materials or documentation, or asking someone external to the working group. Nearly $50 \%$ of respondents (14) indicated that they would never attempt to modify the software themselves.

\section{USING THIS INSIGHT IN DEVELOPMENT}

The knowledge gathered about current and potential users of OMERO has informed and guided further activity towards refining requirements and enhancing functionality. The insights described in Section 6 have influenced development activity in different ways:

Diversity of technology used: Gathering an understanding of the types of software used has been critical for a number of reasons, Further investigations into how scientists use a particular application, such as Adobe's Photoshop, have helped inform decisions relating to whether to attempt to replicate the application functionality the scientist uses, or support data exporting in order to most effectively integrate that application into the OMERO image management and analysis workflow. This work has also informed user interface development, in terms of visual metaphors, labelling and terminology, and interaction styles.

Workflow rigidity: Insights into the extent to which scientists followed strict experimental protocols (i.e. sets of instructions for conducting an experiment), and when and why they might deviate from such protocols have helped to inform the development of OMERO's Protocol Editor functionality. Editability of stages of protocols is supported, enabling users to start with a template protocol and document deviations from or adaptations of this as required; although the inertia of encouraging potentially laborious data input for no immediate benefit is still to be fully addressed.

Interpretation of key concepts - 'image' and 'metadata': Gaining an insight of what scientists understand by an image, and what they understand by metadata has informed user interface design, in terms of labels used, default views of images. One ongoing challenge has been to attempt to preserve the visual memory the scientist had of the image at acquisition time, in rendering the image once imported into OMERO. Given that the pixel data captured by the microscope is displayed to the scientist in a particular way based on the algorithms used by the microscope's software, it can only be considered a representation rather than the correct replication of the imaged sample. Nevertheless any deviations from the scientist's notion of 'correctness' of the same image when displayed in OMERO can lead them to assume that OMERO has somehow 'broken' the image, when in fact the rendering settings applied result in a visually different image - the raw data itself being unchanged. Thus, to a scientist, an image is less a mathematical definition of a collection of data values, and more a visual memory through which they will make a judgment of trust on whether or not OMERO preserves what they consider their 'image'.

Similarly, given diverse interpretations of the term 'metadata', we found it difficult to justify using it as a user interface component label; but given the definitions from the survey, we were able to recommend adjustments to the way OMERO shows data relevant to an image. This data is now grouped according to whether it is automatically acquired by the microscope - exposure settings, date of acquisition, for example; or is later added by the scientist as a comment or tag; or is related to the experiment the image belongs to (for example protocol information, plus organisational information including relationships to other images).

Attitudes to sharing and collaboration: Our work has found evidence to support the existence of reluctance amongst scientists to share experimental data before formal publication of research findings, also identified by De Roure and Goble [5] when discussing the development of a collaborative experiment management system. This has led to a tension within the development team over the effort that should be expended to meet personal user needs, while at the same time presenting opportunities for capitalising on the potential afforded by the quality of data present in OMERO. With images and associated metadata stored on a server, yet highly accessible to anyone who has permission to view it, the potential for remote collaboration and aggregation of data affords new opportunities for discovery; while the persistence of this data over time allows reuse of data for comparison or longitudinal work. All this, though, requires scientists to have access to tools that allow, but not force them to communicate with colleagues and potential collaborators in new ways.

For example, the emergence of support for tagging came from discussions with users over how best OMERO could support organisation of images into logical groups; previously OMERO was limited to a formal pre-defined categorisation structure, but scientists wanted to be able to categorise images on-the-fly when they began to view and analyse them. So categorisation support evolved into a tagging system, enabling user-defined categorisation of images. The next, logical, step is for tagging to support folksonomy creation, where aggregated tagging information can be used to allow scientists to find content of interest that belongs to others, increasing the amount of data that can be analysed and opening up new avenues of discovery. This functionality is now supported by OMERO, but raises questions of permissions and security, as well as a more fundamental question of whether the software can facilitate a cultural shift in terms of a scientist's willingness to make available their data to others before formal publication of their findings.

The need for such a cultural shift is a key factor cited in the apparent lack of success in 'Web 2.0' applications in supporting 
scientific investigation (see for example [4]), and the extent to which OMERO can or should be a catalyst for this shift will remain a core area of focus for the remainder of the Usable Image project.

Dealing with problems with technology: The insights found here provide an underlying reminder of the importance of usability of technology - given the cognitive demands placed on conducting scientific research, the need for simplicity and reliability, for support and efficient error recovery in the design of a system such as OMERO cannot be overstated.

\section{REFLECTION}

Both activities have raised general awareness of OMERO's development at a local and global level, and also of the project's commitment to involving users at all stages of development:

- The ethnographic work has helped to raise user awareness about OMERO - indirectly, as the system played no part in observations - through fostering relationships and trust amongst lab leaders and their scientists, establishing contacts for future participation, and showing evidence of involvement (we are returning to scientists from different labs for feedback, and involvement in further, more focused studies).

- The surveys have identified contacts who can form potential ways into introducing OMERO to new research institutions. While the OME consortium, as an open source software development project has had success in attracting developers from elsewhere to contribute to development, including developers and system administrators working at life science institutions, and commercial biotechnology enterprises, engagement with end users has historically been less successful - partly due to the complexity of installation of OMERO as a client-server system.

Our work has also emphasised the overlap between user-centred design and marketing, especially in an open source software development project where resistance to uptake may be substantial and resources focusing on evangelising uptake are limited. In identifying and involving more users in the UCD process, we realise we must first gain their trust - and that of key gatekeepers such as professors and lab leaders, system administrators - such that they are aware this is a worthwhile activity. By involving these scientists, the aim is that they become champions of the software within their working groups, in a similar way to the 'community champions' of De Roure and Goble [5]. Ongoing activity is looking at how this 'marketing' process can be optimised in a development team with little capacity for non-development related activity; one advance in this area is for each developer in the team to produce a video demonstration of developments as part of every release of the software.

We have also learned to look out for, and manage, potential adverse effects of applying UCD - raised expectations amongst scientists that requests will be immediately acted upon by developers, when sometimes a 'simple request' is a complex programming challenge. We have seen the impact of enhanced user awareness amongst the development team, and a resultant enhanced willingness for the developers to meet directly with amore diverse group of users than their immediate colleagues in the lab. While the Usable Image team have seen ourselves to some extent as an 'airbag' between user and developer, we accept that more direct contact from developers, who are looking for specific questions relating to requirements, can in some cases be more efficient than a request to us to ask the question on their behalf.

\section{FUTURE WORK}

The insights generated from these activities have helped us understand the diversities and similarities in scientific workflow amongst different scientists, with a particular - but not exclusive focus on scientists who work with light microscopy images. This, along with recognised biases in sampling introduced through limitations of the survey recruitment approach, means that there may be some limitations in the extent to which our insights can be generalised across other scientific disciplines. However, the goals of OMERO as a data management and collaboration tool are not confined to supporting scientists working with light microscopy images, and therefore we intend to extend the scope of our studies to scientists working in other fields and with other materials, and who could potentially benefit from a tool such as OMERO.

Therefore, our work has been extended beyond a basic desire to enhance the usability of OMERO to existing users to contributing to efforts to widen appeal to OMERO to people who do not currently use the system. Evaluating the efficacy of our approach is important, but will present challenges - while traditional usability evaluation methods [14] can generate data that allows mapping improvement of quality over time, we are also investigating other ways of demonstrating that a multi-faceted approach can lead to enhanced developer empathy and understanding, and thus better quality and more widely used software.

A further area of ongoing study is to capitalise on the rich array of contextual data gathered through our various activities to formulate an information ecology [12] of the scientific laboratory, and to explore ways of making this more holistic view of the working environment available to developers as a design tool in an effective and useful way.

\section{ACKNOWLEDGMENTS}

We are indebted to Dr Xinyi Jiang, who provided us with a rich collection of ethnographic material for the project to use, and to all scientists who have contributed to our work, either through completing a survey or consenting to be the subject of our ethnographic observations. We also thank the OMERO development team for their support and responsiveness to our work; and to the reviewers of the original draft of this paper for their helpful comments. Usable Image is funded by a grant from the UK Environmental and Physical Sciences Research Council (EPSRC); development of OMERO is supported by the Wellcome Trust and Biotechnology and Biological Sciences Research Council (BBSRC).

\section{REFERENCES}

[1] Manifesto for Agile Software Development: http://agilemanifesto.org/

[2] Baxter S., Day S., Fetrow J. and Reisinger S. 2006 Scientific Software Development Is Not an Oxymoron. PLoS Comput Biol 2(9): e87. DOI: doi:10.1371/journal.pcbi.0020087 
[3] Buckingham S. 2008 Scientific software: seeing the SNPs between us. Nature Methods 5. 903 - 908. DOI=doi:10.1038/nmeth1008-903

[4] Crotty D. 2008 Why Web 2.0 is failing in Biology. http://www.cshblogs.org/cshprotocols/2008/02/14/why-web20-is-failing-in-biology/

[5] De Roure D. and Goble C. 2009 Software Design for Empowering Scientists. IEEE Software 26(1) Jan/Feb 2009. IEEE, 88-95.

[6] Goldberg I., Allan C., Burel J., Creager D., Falconi A., Hochheiser H., Johnston J., Mellen J., Sorger P. and Swedlow J. 2005 The Open Microscopy Environment (OME) Data Model and XML file: open tools for informatics and quantitative analysis in biological imaging. Genome Biol. 2005;6(5) DOI=doi:10.1186/gb-2005-6-5-r47

[7] Gould J., Boies S. and Ukelson J. 1997 How to design Usable Systems. In: Helander M, Landauer T and Prabhu P (eds) Handbook of Human-Computer Interaction. Elsevier, 231-254.

[8] Javahery H., Seffa A. and Radhakrishnan T. 2004 Beyond Power: Making Bioinformatics User-Centred. Comm. ACM, 47(11). New York:ACM Press, 58-63 DOI= doi.acm.org/10.1145/1029496.1029527

[9] Letondal C. and Mackay W. 2004 Participatory Programming and the Scope of Mutual Responsibility: Balancing Scientific, Design and Software Commitment. Proc. Participatory Design Conference 2004 (PDC 04). New York: ACM Press. 31-41. DOI= http://doi.acm.org/10.1145/1011870.1011875
[10] Macaulay C., Benyon D. and Crerar A. 2000 Ethnography, Theory and Design: From Intuition to Insight. Int. J HumanComputer Studies 53(1). Duluth MI: Academic Press, 35-60. DOI= 10.1006/ijhc. 2000.0376

[11] Macaulay C., Sloan D., Jiang X., Forbes P., Loynton S., Swedlow J. and Gregor P. 2009 Usability and User-centred Design in Scientific Software Development. IEEE Software 26(1), Jan/Feb 2009. IEEE: 96-102.

[12] Nardi B. and O’Day V. 1999 Information Ecologies: Using Technology with Heart. Cambridge: MIT Press.

[13] Nichols D. and Twidale M. 2003 The Usability of Open Source Software. First Monday 8(1). http://www.firstmonday.org/Issues/issue8_1/nichols

[14] Nielsen, J., \& Mack, R. L. (Eds.) 1994 Usability inspection methods. New York, NY: John Wiley \& Sons.

[15] Sanders R. and Kelly D. 2008 The Challenge of Testing Scientific Software. Proc Conference of the Association of Software Testing, (Toronto, Canada, July 14-16 2008) CAST 2008.

http://www.associationforsoftwaretesting.org/documents/cast 08/CAST2008_Proceedings.pdf

[16] schraefel m., Hughes G., Mills H., Smith G., Payne T. and Frey J. 2004 Breaking the Book: Translating the Chemistry Lab Book into a Pervasive Computing Lab Environment. Proc. ACM Conf. Human Factors in Computing Systems (Vienna, Austria, 24-29 April 2004) CHI 04. ACM Press, New York: 25-32. DOI= 